\title{
DRIFT-COMPRESSIONAL WAVES PROPAGATING IN THE DIRECTION OF ENERGETIC ELECTRON DRIFT IN THE MAGNETOSPHERE
}

\author{
D.V. Kostarev \\ Institute of Solar-Terrestrial Physics SB RAS, \\ Irkutsk,Russia,kostarev@iszf.irk.ru
}

\author{
P.N. Mager \\ Institute of Solar-Terrestrial Physics SB RAS, \\ Irkutsk,Russia,p.mager@iszf.irk.ru
}

\begin{abstract}
As shown within the gyrokinetic framework, drift-compressional waves can propagate in the magnetosphere in the direction of energetic electron drift. The plasma is assumed to be composed of cold particles with an admixture of hot protons with a Maxwell distribution and electrons with an inverted distribution. The conditions of existence of such waves and their intensification due to resonance interaction with energetic electrons (drift instability) have been de-
\end{abstract}

termined. The results can be helpful in interpreting observation of wave phenomena in the magnetosphere with frequencies in the range of geomagnetic pulsations Pc5 and below.

Keywords: magnetosphere, ULF waves, waveparticle interaction.

\section{INTRODUCTION}

Magnetospheric plasma exhibits a wide spectrum of ultralow-frequency (ULF) oscillations, also called geomagnetic pulsations. They are identified with magnetohydrodynamic (MHD) waves. From both observational and theoretical viewpoints, they can be divided into two large groups: waves with small values of azimuthal wave number $m$ and waves with large values of $m$ [Yeoman et al., 1992; Leonovich, Mazur, 1993; Fenrich et al., 1995].

Waves with small $\mathrm{m}$ values have predominantly toroidal polarization, i.e. the wave magnetic field lines oscillate in the azimuthal direction. They have large azimuthal dimensions and can be observed with groundbased magnetometers. They are sometimes called transverse large-scale oscillations. Usually these waves are identified with Alfvén modes whose sources are located in the outer magnetosphere. A fast magnetosonic (FMS) wave, originated from the magnetopause or the solar wind, is assumed to propagate into the inner magnetosphere, where it generates an Alfvén mode in the field line resonance, at which the FMS wave frequency coincides with the local eigenfrequency of the field line resonance [Chen, Hasegawa, 1974; Southwood, 1974].

Waves with large values of the azimuthal wave number have largely poloidal polarization, i.e. the wave magnetic field lines oscillate in the radial direction. They have small azimuthal dimensions and represent more local events than oscillations with small $m$. These waves can be called transversely small-scale. They are usually identified with poloidal Alfvén modes. The waves are believed to arise from processes occurring in the inner magnetosphere. Due to ionospheric shielding, geomagnetic pulsations with large azimuthal wave numbers can be experimentally studied only with the aid of artificial Earth satellites or radars.

Among the waves with large $m$ in the Pc5 range there is a group of storm-time compressional oscillations whose frequencies can be much lower than the fundamental frequency of Alfvén resonance in the given $\mathrm{L}$ shell. Such oscillations can be recorded both by satellites [Barfield, McPherron, 1972] and by groundbased radars [Allan et al., 1982].

There is still no consensus on the physical nature of the storm-time compressional Pc5 pulsations. According to the magnetohydrodynamic theory, this should be the lowest-frequency mode - the slow magnetosonic mode. However, it is not entirely clear whether the MHD approximation is valid for describing oscillations with frequencies much lower than the Alfvén range in a collisionless plasma since in this case bounce frequencies should be taken into account, which can correctly be done only with the kinetic approach [Hurricane et al., 1994 ]. Storm-time Pc5 pulsations are sometimes associated with drift mirror modes, which are kinetic in nature [Kremser et al., 1981, Pokhotelov et al., 2001]. Still, satisfying mirror instability conditions requires strong temperature anisotropy in magnetospheric plasma.

In our opinion, drift-compressional modes are best suited for interpreting most storm-time compressional Pc5 pulsations. They are the most common compressional modes in kinetics since they demand for their existence only finite plasma pressure and inhomogeneity across magnetic shells. In this case, the instability of the drift-compressional modes can arise from spatial gradients of hot plasma density [Crabtree et al., 2003; Klimushkin, Mager, 2011], inverted distribution of hot proton energy [Mager et al., 2013], or from the coupling with the Alfvén mode due to 
magnetic field line curvature [Klimushkin et al., 2012]. By the inverted distribution is meant the nonmonotonic velocity distribution with its maximum in the highenergy part of spectrum, by analogy with [Hughes et al., 1978]. A characteristic feature of drift-compressional waves is the dependence of their frequency on the azimuthal wave number. A similar behavior pattern has been found in radar data [Mager et al., 2015; Chelpanov et al., 2016].

It has been shown that the drift-compressional modes propagating in the direction of the drift of highenergy protons interact resonantly with them. As temperature increases and particle density decreases with distance away from Earth, this can cause instability and a spontaneous increase in waves whose phase velocity direction coincides with the proton drift direction [Mager et al., 2013]. The instability threshold is lowered if the proton distribution function is inverted. However, as shown in [James et al., 2013], in some cases there are waves propagating in the opposite direction, i.e. in the electron drift direction. Therefore, in this paper we study the situation in which the wave propagates in the electron drift direction. Here, we assume that there are hot protons and electrons in plasma, the latter having an inverted energy distribution.

\section{MODEL OF ENVIRONMENT AND BASIC EQUATIONS}

We use an axially symmetric model of the magnetosphere, which takes into account the field line curvature and the background plasma inhomogeneity across magnetic shells and along field lines. To do this, we introduce an orthogonal coordinate system $\left\{x^{1}, x^{2}\right.$, $\left.x^{3}\right\}$ such that the coordinate $x^{1}$ coincides with the magnetic shells, $x^{2}$ indicates a magnetic field line (azimuthal coordinate), and $x^{3}$ is a field line point; $g_{1}$, $g_{2}$, and $g_{3}$ are respective coordinates of a metric tensor; $d l=\sqrt{g_{3} d x^{3}}$ is the length element along the field line [Leonovich, Mazur, 1989]. Taking into account the curvature and field-aligned inhomogeneity of the magnetic field makes particles trapped in the magnetosphere.

We consider plasma with an admixture of hot protons and electrons. Since the contribution of cold particles to the total plasma pressure is small, we consider only the contribution of hot particles. Here we assume that the protons have a Maxwell energy distribution:

$$
F_{\mathrm{p}}=\frac{n_{\mathrm{p}}}{\left(2 \pi \varepsilon_{0_{\mathrm{p}}}\right)^{\frac{3}{2}}} \exp \left(-\frac{\varepsilon}{\varepsilon_{0_{\mathrm{p}}}}\right),
$$

and the hot electrons have an inverted distribution and are modeled by the following function:

$$
\begin{aligned}
& F_{\mathrm{e}}=\frac{n_{\mathrm{e}}}{4 \sqrt{2} \pi \Gamma(S+3 / 2)\left(\varepsilon_{0_{\mathrm{e}}}\right)^{S+\frac{3}{2}}}\left(\frac{\varepsilon}{\varepsilon_{0_{\mathrm{e}}}}\right)^{S} \times \\
& \times \exp \left(-\frac{\varepsilon}{\varepsilon_{0_{\mathrm{e}}}}\right) .
\end{aligned}
$$

Here, $n_{\mathrm{p}}$ and $n_{\mathrm{e}}$ are proton and electron densities respectively; $\varepsilon=v^{2} / 2$ is the mass-normalized particle energy; $V$ is the particle velocity; $S$ is the positive integer; $G(\ldots)$ is the gamma function; $\varepsilon_{0_{\mathrm{p}}}$ and $\varepsilon_{0_{\mathrm{e}}}$ are the parameters proportional to the squared thermal velocity of particles. Hereafter, the indices " $p$ " and "e" are the proton and electron variables respectively.

Note that with $S=0$, electron distribution function (2) becomes the Maxwell distribution. For $S>0$, electrons have the mean particle energy $\bar{\varepsilon}_{\mathrm{e}}=(S+3 / 2) \varepsilon_{0_{\mathrm{e}}}$ and energy at maximum $\varepsilon_{\mathrm{e}_{\max }}=S \varepsilon_{0_{\mathrm{e}}}$.

We employ the axially symmetric model of the magnetosphere. The dependence of disturbed parameters on time and coordinates is represented as

$$
\exp \left[-i \omega t+i \int k_{1}\left(x^{1}\right) d x^{1}+i k_{2} x^{2}\right],
$$

where $\omega$ is the wave frequency; $k_{1}$ and $k_{2}$ are the radial and azimuthal wave vector components respectively.

Plasma oscillations with a frequency less than the gyrofrequency of plasma particles can be considered within a gyrokinetic framework in the WKB approximation [Chen, Hasegawa, 1991]. In the approximation in which the wave frequency is much less than the bounce frequency of particles, an equation describing the drift-compressional mode can be derived from the gyrokinetic equations presented in [Chen, Hasegawa, 1991]. However, unlike previous works [Crabtree et al., 2003, Klimushkin, Mager, 2011; Mager et al., 2013] which took a wave whose direction of propagation coincided with the proton direction $k_{2}<0$, i.e. to the west, we consider the case where the wave propagates east, in the electron drift direction $k_{2}>0$.

Thus, we study the electron wave-particle resonance: in the equation for the drift-compressional mode, the sign in the resonant denominator of the term describing the contribution of hot protons changes to positive, i.e. no wave-particle resonance exists for protons, but it exists for electrons, and the sign in their resonant denominator becomes negative:

$$
\begin{aligned}
& b_{\|}(l)=4 \pi m_{\mathrm{p}}\left\langle\frac{\hat{Q}_{\mathrm{p}} F_{\mathrm{p}}}{\omega+\bar{\omega}_{\mathrm{d}_{\mathrm{p}}}} \mu \overline{\left(\mu b_{\|}(l)\right)}\right\rangle+ \\
& +4 \pi m_{\mathrm{e}}\left\langle\frac{\hat{Q}_{\mathrm{e}} F_{\mathrm{e}}}{\omega-\omega_{\mathrm{d}_{\mathrm{e}}}} \mu \overline{(\mu b(l))}\right\rangle .
\end{aligned}
$$


Here $b_{\|}$is the longitudinal component of the wave magnetic field; $|l|$ is the distance along the field line from the magnetic equator to a given point; $\langle\ldots\rangle$ is the velocity space integral:

$$
\langle\ldots\rangle=4 \pi \int(\ldots) \frac{B}{\left|v_{\|}\right|} d \mu d \varepsilon
$$

$\overline{(\ldots)}$ is the average over the bounce period $\tau_{\mathrm{b}}$ :

$$
\overline{(\ldots)}=\frac{2}{\tau_{\mathrm{b}}} \int_{-l_{0}}^{l_{0}}(\ldots)\left|v_{\|}\right|^{-1} d l ; \tau_{\mathrm{b}}=2 \int_{-l_{0}}^{l_{0}}\left|v_{\|}\right|^{-1} d l,
$$

where $\pm l_{0}$ denotes the reflection points on the ionosphere for particles with energy $\varepsilon$ and magnetic moment $\mu=v_{\perp}^{2} /(2 B) ; m_{\mathrm{p}}$ and $m_{\mathrm{e}}$ are the proton and electron masses respectively; $\omega_{d_{p, e}}$ is the drift frequency:

$$
\omega_{\mathrm{d}_{\mathrm{p}, \mathrm{e}}}=\left|\vec{k}_{\perp} \vec{V}_{\mathrm{d}_{\mathrm{p}, \mathrm{e}}}\right|=\left|\frac{k_{2}}{\omega_{c_{\mathrm{p}, \mathrm{e}}} \sqrt{g_{2}}}\left(\frac{1}{\sqrt{g_{1}}} \frac{B^{\prime}}{2 B} v_{\perp}^{2}-\frac{v_{\|}^{2}}{R}\right)\right|,
$$

where $v_{\|}$and $v_{\infty}$ are the longitudinal and transverse particle velocities; $\vec{V}_{\mathrm{d}}$ is the particle magnetic drift velocity; $\omega_{c_{\mathrm{p}, \mathrm{e}}}=e B / m_{\mathrm{p}, \mathrm{e}}$ is the gyrofrequency; $R$ is the magnetic field curvature radius. The operator $\hat{Q}$ is determined as follows:

$$
\hat{Q}_{\mathrm{p}, \mathrm{e}}=\omega \frac{\partial}{\partial \varepsilon} \pm \frac{k_{2}}{\omega_{c_{\mathrm{p}, \mathrm{e}}} \sqrt{g_{2}}} \frac{1}{\sqrt{g_{1}}} \frac{\partial}{\partial x^{1}} .
$$

Here, the sign "+" indicates protons; the sign "--" marks electrons.

After changing variables $\varepsilon, \mu \rightarrow \xi, \quad \lambda$ such that $\lambda=\sin ^{2} \alpha=\mu B_{0} / \varepsilon, \quad \alpha$ is the pitch angle, $B_{0}$ is the magnetic field at the equator, $\xi=\sqrt{\varepsilon / \varepsilon_{0}}$, equation (3) can be represented as

$b_{\|}(l)=\int_{0}^{\frac{B_{0}}{B(l)}} d \lambda \int_{0}^{l_{0}(\lambda)} d l^{\prime} \frac{B(l)}{B_{0}} \frac{\lambda^{2} \Lambda(\omega, \lambda)}{u(l, \lambda) u\left(l^{\prime}, \lambda\right)} b\left(l^{\prime}\right)$,

where

$$
\begin{aligned}
& u(l, \lambda)=\sqrt{1-\lambda B(l) / B_{0}}, \\
& \Lambda(\omega, \lambda)=\frac{\beta_{0_{\mathrm{p}}}}{L_{\mathrm{b}_{\mathrm{p}}}} I_{\mathrm{p}}(\omega, \lambda)+\frac{3}{2} \frac{\beta_{0_{\mathrm{e}}}}{L_{\mathrm{b}_{\mathrm{e}}}} \frac{\sqrt{\pi}}{\Gamma(S+5 / 2)} I_{\mathrm{e}}(\omega, \lambda), \\
& I_{\mathrm{p}}(\omega, \lambda)=\frac{1}{\sqrt{\pi}} \int_{0}^{+\infty} d \xi_{\mathrm{p}}^{2} \frac{\xi_{\mathrm{p}}^{5} e^{\xi_{\mathrm{p}}^{2}}}{\xi_{\mathrm{p}}^{2}+\omega / \Omega_{\mathrm{d}_{\mathrm{p}}}} \times \\
& \times\left[\frac{\omega_{n_{\mathrm{p}}}^{*}}{\Omega_{\mathrm{d}_{\mathrm{p}}}}-\frac{\omega}{\Omega_{\mathrm{d}_{\mathrm{p}}}}-\left(\frac{3}{2}-\xi_{p}^{2}\right) \frac{\omega_{\varepsilon_{\mathrm{p}}}^{*}}{\Omega_{\mathrm{d}_{\mathrm{p}}}}\right],
\end{aligned}
$$

$$
\begin{aligned}
& I_{\mathrm{e}}(\omega, \lambda)=\frac{1}{\sqrt{\pi}} \int_{-\infty}^{+\infty} d \xi_{\mathrm{e}} \frac{\xi_{\mathrm{e}}^{5+2 S} e^{-\xi_{\mathrm{e}}^{2}}}{\xi_{\mathrm{e}}-\sqrt{\omega / \Omega_{\mathrm{d}_{\mathrm{e}}}}} \times \\
& \times\left[\frac{\omega}{\Omega_{\mathrm{d}_{\mathrm{e}}}}\left(1-\frac{S}{\xi_{\mathrm{e}}^{2}}\right)-\frac{\omega_{n_{\mathrm{e}}}^{*}}{\Omega_{\mathrm{d}_{\mathrm{e}}}}+\left(S+\frac{3}{2}-\xi_{\mathrm{e}}^{2}\right) \frac{\omega_{\varepsilon_{\mathrm{e}}}^{*}}{\Omega_{\mathrm{d}_{\mathrm{e}}}}\right] .
\end{aligned}
$$

The variables

$$
\begin{aligned}
& \omega_{n_{\mathrm{p}}}^{*}=\frac{1}{\sqrt{g_{1}}} \frac{k_{2} \varepsilon_{0}}{\omega_{c_{\mathrm{p}}} \sqrt{g_{2}}} \frac{n_{\mathrm{p}}^{\prime}}{n_{\mathrm{p}}}, \\
& \omega_{n_{\mathrm{e}}}^{*}=-\frac{1}{\sqrt{g_{1}}} \frac{k_{2} \varepsilon_{0}}{\omega_{c_{\mathrm{e}}} \sqrt{g_{2}}} \frac{n_{\mathrm{e}}^{\prime}}{n_{\mathrm{e}}}
\end{aligned}
$$

and

$$
\begin{aligned}
& \omega_{\varepsilon_{\mathrm{p}}}^{*}=\frac{1}{\sqrt{g_{1}}} \frac{k_{2} \varepsilon_{0}}{\omega_{c_{\mathrm{p}}} \sqrt{g_{2}}} \frac{\varepsilon_{0_{\mathrm{p}}}^{\prime}}{\varepsilon_{0_{\mathrm{e}}}}, \\
& \omega_{\varepsilon_{\mathrm{e}}}^{*}=-\frac{1}{\sqrt{g_{1}}} \frac{k_{2} \varepsilon_{0}}{\omega_{c_{\mathrm{e}}} \sqrt{g_{2}}} \frac{\varepsilon_{0_{\mathrm{e}}}^{\prime}}{\varepsilon_{0_{\mathrm{e}}}}
\end{aligned}
$$

correspond to the diamagnetic frequencies $\omega^{*}=\vec{k}_{\perp} \vec{V}^{*}$, where $\vec{V}^{*}$ is the velocity of the particle diamagnetic drift driven by the radial gradient of plasma density or temperature; $\Omega_{\mathrm{d}}=\bar{\omega}_{\mathrm{d}} \varepsilon_{0} / \varepsilon$ is the bounce-periodaveraged drift frequency of particles with energy $\varepsilon_{0}$; $\beta_{0}$ is the parameter describing the ratio of plasma pressure to magnetic pressure at the equator; $L_{\mathrm{b}}$ is the particle path length for the bounce period:

$$
L_{\mathrm{b}}=v \tau_{\mathrm{b}}=4 \int_{0}^{l_{0}} u(l, \lambda)^{-1} d l .
$$

In the dipole magnetic field, $L_{\mathrm{b}}$ and $\Omega_{\mathrm{d}}$ weakly depend on $\lambda: \quad L_{\mathrm{b}} \sim 1.3-0.56 \sqrt{\lambda}$ and $\Omega_{\mathrm{d}} \sim 0.35+0.15 \sqrt{\lambda}$ [Hamlit et al., 1961]. Therefore, we assume that $\Lambda$ is independent of $\lambda$. Then, in Equation (4) we can take $\Lambda$ outside the integrals. Next, we can carry out a number of transformations, as in [Mager et al., 2013], and obtain a second-kind homogeneous Fredholm integral equation with a symmetric kernel. This equation can be solved numerically. In this case, we get sets of eigenfunctions $b_{N}$ and eigenvalues $\Lambda_{N}$ in the integral equation, which determine the field-aligned structure and eigenfrequencies of drift-compressional modes. As shown in [Mager et al., 2013], drift-compressional modes are localized sin the vicinity of the geomagnetic equator. This agrees with satellite data on compressional Pc5 pulsations [Higuchi, Kokubun, 1988].

\section{EIGENFREQUENCIES AND INSTABILITY CONDITIONS}

The eigenfrequencies are determined from the dispersion relation $\Lambda(\omega)=\Lambda_{N}$, i.e. 


$$
\Lambda_{N}=\frac{\beta_{0_{\mathrm{p}}}}{L_{\mathrm{b}_{\mathrm{p}}}} I_{\mathrm{p}}(\omega)+\frac{3}{2} \frac{\beta_{0_{\mathrm{e}}}}{L_{\mathrm{b}_{\mathrm{e}}}} \frac{\sqrt{\pi}}{\Gamma(S+5 / 2)} I_{\mathrm{e}}(\omega) .
$$

After taking the integrals, we have

$$
\Lambda_{N}=\frac{\beta_{\mathrm{p}}}{L_{\mathrm{b}_{\mathrm{p}}}} f_{\mathrm{p}}(\omega)+\frac{3}{2} \frac{\beta_{\mathrm{e}}}{L_{\mathrm{b}_{\mathrm{e}}}} f_{\mathrm{e}}(\omega) \text {. }
$$

Here

$$
\begin{aligned}
& f_{\mathrm{e}}(\omega)=\frac{\omega}{\Omega_{\mathrm{d}_{\mathrm{e}}}}\left(1-\frac{\omega_{s_{\mathrm{e}}}^{*}}{\Omega_{\mathrm{d}_{\mathrm{e}}}}\right)-\left(\frac{\omega_{n_{\mathrm{e}}}^{*}}{\Omega_{\mathrm{d}_{\mathrm{e}}}}+\frac{\omega_{\mathrm{s}_{\mathrm{e}}}^{*}}{\Omega_{\mathrm{d}_{\mathrm{e}}}}\right)+ \\
& +\frac{\omega}{\Omega_{\mathrm{d}_{\mathrm{e}}}}\left[\left(\frac{\omega}{\Omega_{\mathrm{d}_{\mathrm{e}}}}-S\right)\left(1-\frac{\omega_{\varepsilon_{\mathrm{e}}}^{*}}{\Omega_{\mathrm{d}_{\mathrm{e}}}}\right)-\frac{\omega_{n_{\mathrm{e}}}^{*}}{\Omega_{\mathrm{d}_{\mathrm{e}}}}+\frac{3}{2} \frac{\omega_{\varepsilon_{\mathrm{e}}}^{*}}{\Omega_{\mathrm{d}_{\mathrm{e}}}}\right] \times \\
& \times \frac{\sqrt{\pi}}{\Gamma(S+5 / 2)}\left[\sum_{m=0}^{S+1} \frac{\Gamma(m+1 / 2)}{\sqrt{\pi}}\left(\frac{\omega}{\Omega_{\mathrm{d}_{\mathrm{e}}}}\right)^{S+1-m}+\right. \\
& \left.+\left(\frac{\omega}{\Omega_{\mathrm{d}_{\mathrm{e}}}}\right)^{S+\frac{3}{2}} Z\left(\sqrt{\frac{\omega}{\Omega_{\mathrm{d}_{\mathrm{e}}}}}\right)\right],
\end{aligned}
$$

where $Z\left(\sqrt{\frac{\omega}{\Omega_{\mathrm{d}_{\mathrm{e}}}}}\right)$ is the plasma dispersion function

[Walker, 2005],

$$
\begin{aligned}
& Z\left(\sqrt{\frac{\omega}{\Omega_{\mathrm{d}_{\mathrm{e}}}}}\right)=\frac{1}{\sqrt{\pi}} \int_{-\infty}^{+\infty} \frac{e^{-t^{2}}}{t-\sqrt{\omega / \Omega_{\mathrm{d}_{\mathrm{e}}}}} d t . \\
& f_{\mathrm{p}}(\omega)=\frac{15}{8} \frac{\omega_{\varepsilon_{\mathrm{p}}}^{*}}{\Omega_{\mathrm{d}_{\mathrm{p}}}}+ \\
& +\left(\frac{\omega_{n_{\mathrm{p}}}^{*}}{\Omega_{\mathrm{d}_{\mathrm{p}}}}-\frac{3}{2} \frac{\omega_{\varepsilon_{\mathrm{p}}}^{*}}{\Omega_{\mathrm{d}_{\mathrm{p}}}}-\frac{\omega}{\Omega_{\mathrm{d}_{\mathrm{p}}}}-\frac{\omega_{\varepsilon_{\mathrm{p}}}^{*}}{\Omega_{\mathrm{d}_{\mathrm{p}}}} \frac{\omega}{\Omega_{\mathrm{d}_{\mathrm{p}}}}\right) \times \\
& \times\left(\frac{3}{4}-\frac{1}{2} \frac{\omega}{\Omega_{\mathrm{d}_{\mathrm{p}}}}+\left(\frac{\omega}{\Omega_{\mathrm{d}_{\mathrm{p}}}}\right)^{2}-\left(\frac{\omega}{\Omega_{\mathrm{d}_{\mathrm{p}}}}\right)^{3} Z^{+}\left(\sqrt{\frac{\omega}{\Omega_{\mathrm{d}_{\mathrm{p}}}}}\right)\right), \\
& Z^{+}\left(\sqrt{\frac{\omega}{\Omega_{\mathrm{d}_{\mathrm{p}}}}}\right)=\frac{2}{\sqrt{\pi}} \int_{0}^{+\infty} \frac{e^{-t^{2}}}{t^{2}+\omega / \Omega_{\mathrm{d}_{\mathrm{p}}}} d t= \\
& =\sqrt{\pi} \sqrt{\frac{\Omega_{\mathrm{d}_{\mathrm{p}}}}{\omega}} e^{\frac{\omega}{\Omega_{\mathrm{d}_{\mathrm{p}}}}} \operatorname{Erfc}_{c}\left(\sqrt{\frac{\omega}{\Omega_{\mathrm{d}_{\mathrm{p}}}}}\right),
\end{aligned}
$$

where $\operatorname{Erfc}\left(\sqrt{\frac{\omega}{\Omega_{\mathrm{d}_{\mathrm{p}}}}}\right)$ is the complementary error function:

$$
\operatorname{Erfc}\left(\sqrt{\frac{\omega}{\Omega_{\mathrm{d}_{\mathrm{p}}}}}\right)=\frac{2}{\sqrt{\pi}} \int_{\sqrt{\omega / \Omega_{\mathrm{d}_{\mathrm{p}}}}}^{+\infty} e^{-t^{2}} d t .
$$

Now, to find the eigenfrequencies and possible instability increments, we represent the wave frequency as $\omega=\omega_{0}+i \gamma$. We consider the real part of frequency as being much larger than the imaginary part: $\omega_{0} \gg \gamma$.

Suppose $\alpha=\Omega_{\mathrm{d}_{\mathrm{e}}} / \Omega_{\mathrm{d}_{\mathrm{p}}}$, then, by changing variables in Expression (8), we can rewrite the dispersion relation as

$$
\frac{L_{\mathrm{b}_{\mathrm{p}}}}{\beta_{\mathrm{p}}} \Lambda_{N}=f_{\mathrm{p}}\left(\alpha \frac{\omega}{\Omega_{\mathrm{d}_{\mathrm{e}}}}\right)+\frac{3}{2} \frac{\beta_{\mathrm{e}}}{\beta_{\mathrm{p}}} f_{\mathrm{e}}\left(\frac{\omega}{\Omega_{\mathrm{d}_{\mathrm{e}}}}\right) .
$$

The parameter $\alpha$ represents the ratio of electron energy to proton energy: $\alpha=m_{\mathrm{e}} \varepsilon_{0_{\mathrm{e}}} / m_{\mathrm{p}} \varepsilon_{0_{\mathrm{p}}}$. For simplicity, we take hot proton and electron densities, which contribute to plasma pressure, as being equal. Then, $\alpha$ can be represented as

$$
\alpha=\frac{3}{2} \frac{\beta_{\mathrm{e}}}{\beta_{\mathrm{p}}} \frac{1}{(S+3 / 2)} .
$$

If we consider the case close to the ring current conditions, then we can say that the proton energy is much higher than the electron energy, i.e. $\alpha \ll 1$ and, accordingly, $\beta_{\mathrm{e}} / \beta_{\mathrm{p}} \ll 1$. Then, we can expand the function $f_{\mathrm{p}}\left(\alpha \omega / \Omega_{\mathrm{d}_{\mathrm{e}}}\right)$ of Expression (11) in the small parameter $\alpha$. Neglecting all terms such that the power of $\alpha$ is greater than 1 , we get

$$
\begin{aligned}
& f_{\mathrm{p}}\left(\alpha \frac{\omega}{\Omega_{\mathrm{d}_{\mathrm{e}}}}\right)=\frac{3}{4}\left(\frac{\omega_{s_{\mathrm{p}}}^{*}}{\Omega_{\mathrm{d}_{\mathrm{p}}}}+\frac{\omega_{n_{\mathrm{p}}}^{*}}{\Omega_{\mathrm{d}_{\mathrm{p}}}}\right)- \\
& -\frac{1}{2} \alpha \frac{\omega}{\Omega_{\mathrm{d}_{\mathrm{e}}}}\left(\frac{3}{2}+\frac{\omega_{n_{\mathrm{p}}}^{*}}{\Omega_{\mathrm{d}_{\mathrm{p}}}}\right) .
\end{aligned}
$$

To find an analytical solution, we expand $f_{\mathrm{e}}\left(\omega / \Omega_{\mathrm{d}_{\mathrm{e}}}\right)$ in the small parameter for two extreme cases: when the eigenfrequency is much less than $\left(\omega / \Omega_{d_{e}} \ll 1\right)$ and much greater than $\left(\omega / \Omega_{d_{e}} \gg 1\right)$ of the electron drift frequency.

\section{EIGENFREQUENCY MUCH LESS THAN ELECTRON DRIFT FREQUENCY: $\boldsymbol{\omega} / \mathbf{\Omega}_{\mathrm{d}_{\mathrm{e}}}=\mathbf{1}$}

If we expand $Z\left(\sqrt{\omega / \Omega_{\mathrm{d}_{\mathrm{e}}}}\right)$ in the small parameter $\omega / \Omega_{\mathrm{d}_{\mathrm{e}}} \ll 1$ in $f_{\mathrm{e}}\left(\omega / \Omega_{\mathrm{d}_{\mathrm{e}}}\right)$ and neglect all terms where the power of $\omega / \Omega_{d_{e}}$ is 2 or more, considering them small, we derive the expression 


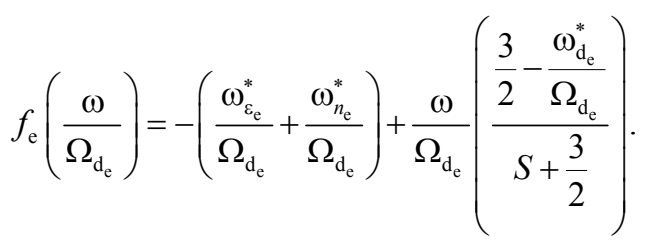

Then, for the case when the wave eigenfrequency is much less than the electron magnetic drift frequency, and the proton energy is much greater than the electron energy, the dispersion relation takes the form

$$
\begin{aligned}
& \frac{L_{\mathrm{b}_{\mathrm{p}}}}{\beta_{\mathrm{p}}} \Lambda_{N}=\frac{3}{4}\left(\frac{\omega_{\varepsilon_{\mathrm{p}}}^{*}}{\Omega_{\mathrm{d}_{\mathrm{p}}}}+\frac{\omega_{n_{\mathrm{p}}}^{*}}{\Omega_{\mathrm{d}_{\mathrm{p}}}}\right)-\frac{1}{2} \alpha \frac{\omega}{\Omega_{\mathrm{d}_{\mathrm{e}}}}\left(\frac{3}{2}+\frac{\omega_{n_{\mathrm{p}}}^{*}}{\Omega_{\mathrm{d}_{\mathrm{p}}}}\right)- \\
& -\frac{3}{2} \frac{\beta_{\mathrm{e}}}{\beta_{\mathrm{p}}}\left[\left(\frac{\omega_{\varepsilon_{\mathrm{e}}}^{*}}{\Omega_{\mathrm{d}_{\mathrm{e}}}}+\frac{\omega_{n_{\mathrm{e}}}^{*}}{\Omega_{\mathrm{d}_{\mathrm{e}}}}\right)-\frac{\omega}{\Omega_{\mathrm{d}_{\mathrm{e}}}}\left(\frac{\frac{3}{2}-\frac{\omega_{n_{\mathrm{e}}}^{*}}{\Omega_{\mathrm{d}_{\mathrm{e}}}}}{S+\frac{3}{2}}\right)\right] .
\end{aligned}
$$

From (13), we derive the eigenfrequency expression:

$$
\times \frac{\frac{L_{0}=\Omega_{\mathrm{d}_{\mathrm{p}}}}{\beta_{\mathrm{p}}} \Lambda_{N}-\frac{3}{4}\left(\frac{\omega_{\varepsilon_{\mathrm{p}}}^{*}}{\Omega_{\mathrm{d}_{\mathrm{p}}}}+\frac{\omega_{n_{\mathrm{p}}}^{*}}{\Omega_{\mathrm{d}_{\mathrm{p}}}}\right)+\frac{3}{2} \frac{\beta_{\mathrm{e}}}{\beta_{\mathrm{p}}}\left(\frac{\omega_{\varepsilon_{\mathrm{e}}}^{*}}{\Omega_{\mathrm{d}_{\mathrm{e}}}}+\frac{\omega_{n_{\mathrm{e}}}^{*}}{\Omega_{\mathrm{d}_{\mathrm{e}}}}\right)}{\frac{3}{4}-\frac{\omega_{n_{\mathrm{e}}}^{*}}{\Omega_{\mathrm{d}_{\mathrm{e}}}}-\frac{1}{2} \frac{\omega_{n_{\mathrm{p}}}^{*}}{\Omega_{\mathrm{d}_{\mathrm{p}}}}} .
$$

To find the increment, we calculate the imaginary part of dispersion relation (11), apply the expansion in the small parameter to the plasma dispersion function, and neglect the terms in the denominator, where the power of $\omega / \Omega_{\mathrm{d}_{\mathrm{e}}}$ is 2 and more:

$$
\begin{gathered}
\gamma=-\Omega_{\mathrm{d}_{\mathrm{e}}} \frac{\pi\left(\frac{\omega_{0}}{\Omega_{\mathrm{d}_{\mathrm{e}}}}\right)^{S+5 / 2} e^{-\frac{\omega_{0}}{\Omega_{\mathrm{d}_{\mathrm{e}}}}} \times}{\Gamma(S+3 / 2)} \times \\
\times \frac{\left[\left(\frac{\omega_{0}}{\Omega_{\mathrm{d}_{\mathrm{e}}}}-S\right)\left(1-\frac{\omega_{\varepsilon_{\mathrm{e}}}^{*}}{\Omega_{\mathrm{d}_{\mathrm{e}}}}\right)-\frac{\omega_{n_{\mathrm{e}}}^{*}}{\Omega_{\mathrm{d}_{\mathrm{e}}}}+\frac{3}{2} \frac{\omega_{\varepsilon_{\mathrm{e}}}^{*}}{\Omega_{\mathrm{d}_{\mathrm{e}}}}\right]}{\frac{3}{4}-\frac{\omega_{n_{\mathrm{e}}}^{*}}{\Omega_{\mathrm{d}_{\mathrm{e}}}}-\frac{1}{2} \frac{\omega_{n_{\mathrm{p}}}^{*}}{\Omega_{\mathrm{d}_{\mathrm{p}}}}}
\end{gathered}
$$

From eigenfrequency expression (14) it follows that the wave can exist in plasma without particle temperature and density gradients. Equations (14) and (15) also show that without these gradients the instability occurs only for the inverted distribution $(S \neq 0)$. In this case, $\omega / \Omega_{\mathrm{d}_{\mathrm{e}}}-S<0$, then $V_{\mathrm{ph}}<V_{\mathrm{d}}\left(\varepsilon=\varepsilon_{\mathrm{e}_{\max }}\right)$. Hence, in the absence of plasma gradients the instability can occur if the wave phase velocity is lower than the mean particle magnetic drift velocity in the bump in the inverted distribution.

The wave existence conditions for $\omega / \Omega_{\mathrm{d}_{\mathrm{e}}} \ll 1$ from (14) are as follows:

$$
\begin{aligned}
& \left\{\begin{array}{c}
\frac{3}{4}-\frac{\omega_{n_{\mathrm{e}}}^{*}}{\Omega_{\mathrm{d}_{\mathrm{e}}}}-\frac{1}{2} \frac{\omega_{n_{\mathrm{p}}}^{*}}{\Omega_{\mathrm{d}_{\mathrm{p}}}}>0, \\
\frac{L_{\mathrm{b}_{\mathrm{p}}}}{\beta_{\mathrm{p}}} \Lambda_{N}-\frac{3}{4}\left(\frac{\omega_{\varepsilon_{\mathrm{p}}}^{*}}{\Omega_{\mathrm{d}_{\mathrm{p}}}}+\frac{\omega_{n_{\mathrm{p}}}^{*}}{\Omega_{\mathrm{d}_{\mathrm{p}}}}\right)+\frac{3}{2} \frac{\beta_{\mathrm{e}}}{\beta_{\mathrm{p}}}\left(\frac{\omega_{\varepsilon_{\mathrm{e}}}^{*}}{\Omega_{\mathrm{d}_{\mathrm{e}}}}+\frac{\omega_{n_{\mathrm{e}}}^{*}}{\Omega_{\mathrm{d}_{\mathrm{c}}}}\right)>0
\end{array}\right. \\
& \text { or } \\
& \left\{\begin{array}{c}
\frac{3}{4}-\frac{\omega_{n_{\mathrm{e}}}^{*}}{\Omega_{\mathrm{d}_{\mathrm{e}}}}-\frac{1}{2} \frac{\omega_{n_{\mathrm{p}}}^{*}}{\Omega_{\mathrm{d}_{\mathrm{p}}}}<0, \\
\frac{L_{\mathrm{b}_{\mathrm{p}}}}{\beta_{\mathrm{p}}} \Lambda_{N}-\frac{3}{4}\left(\frac{\omega_{\varepsilon_{\mathrm{p}}}^{*}}{\Omega_{\mathrm{d}_{\mathrm{p}}}}+\frac{\omega_{n_{\mathrm{p}}}^{*}}{\Omega_{\mathrm{d}_{\mathrm{p}}}}\right)+\frac{3}{2} \frac{\beta_{\mathrm{c}}}{\beta_{\mathrm{p}}}\left(\frac{\omega_{\varepsilon_{\mathrm{e}}}^{*}}{\Omega_{\mathrm{d}_{\mathrm{e}}}}+\frac{\omega_{n_{\mathrm{e}}}^{*}}{\Omega_{\mathrm{d}_{\mathrm{e}}}}\right)<0 .
\end{array}\right.
\end{aligned}
$$

The instability $\gamma>0$ takes place for (16.1) with

$$
\left(\frac{\omega_{0}}{\Omega_{\mathrm{d}_{\mathrm{e}}}}-S\right)\left(1-\frac{\omega_{\varepsilon_{\mathrm{e}}}^{*}}{\Omega_{\mathrm{d}_{\mathrm{e}}}}\right)-\frac{\omega_{n_{\mathrm{e}}}^{*}}{\Omega_{\mathrm{d}_{\mathrm{e}}}}+\frac{3}{2} \frac{\omega_{\varepsilon_{\mathrm{e}}}^{*}}{\Omega_{\mathrm{d}_{\mathrm{e}}}}<0
$$

and for (16.2) with

$$
\left(\frac{\omega_{0}}{\Omega_{\mathrm{d}_{\mathrm{e}}}}-S\right)\left(1-\frac{\omega_{\varepsilon_{\mathrm{e}}}^{*}}{\Omega_{\mathrm{d}_{\mathrm{e}}}}\right)-\frac{\omega_{n_{\mathrm{e}}}^{*}}{\Omega_{\mathrm{d}_{\mathrm{e}}}}+\frac{3}{2} \frac{\omega_{\varepsilon_{\mathrm{e}}}^{*}}{\Omega_{\mathrm{d}_{\mathrm{e}}}}<0
$$

In the equatorial parabolic approximation for the dipole magnetic field for the first harmonic $N=1$, we can take that $\Lambda_{1} \approx 0.5 / L$ and $L_{\mathrm{b}_{\mathrm{p}, \mathrm{e}}}=2 \pi \sqrt{2} L$, where $L$ is the distance to the magnetic shell in the equatorial plane [Mager et al., 2013]. Then

$$
\begin{gathered}
\omega_{n_{\mathrm{p}}}^{*}=\frac{k_{2} \varepsilon_{0_{\mathrm{p}}}}{\omega_{c_{\mathrm{p}}} L} \frac{n_{\mathrm{p}}^{\prime}}{n_{\mathrm{p}}}, \omega_{\varepsilon_{\mathrm{p}}}^{*}=\frac{k_{2} \varepsilon_{0_{\mathrm{p}}}}{\omega_{c_{\mathrm{p}}} L} \frac{\varepsilon_{0_{\mathrm{p}}}^{\prime}}{\varepsilon_{0_{\mathrm{p}}}}, \\
\Omega_{\mathrm{d}_{\mathrm{p}}} \sim \frac{3 k_{2} \varepsilon_{0_{\mathrm{p}}}}{\omega_{c_{\mathrm{p}}} L^{2}}, \omega_{n_{\mathrm{e}}}^{*}=-\frac{k_{2} \varepsilon_{0_{\mathrm{e}}}}{\omega_{c_{\mathrm{e}}} L} \frac{n_{\mathrm{e}}^{\prime}}{n_{\mathrm{e}}}, \\
\omega_{\varepsilon_{\mathrm{e}}}^{*}=-\frac{k_{2} \varepsilon_{0_{\mathrm{e}}}}{\omega_{c_{\mathrm{e}}} L} \frac{\varepsilon_{0_{\mathrm{e}}}^{\prime}}{\varepsilon_{0_{\mathrm{e}}}}, \Omega_{\mathrm{d}_{\mathrm{e}}} \sim \frac{3 k_{2} \varepsilon_{0_{\mathrm{e}}}}{\omega_{c_{\mathrm{e}}} L^{2}} .
\end{gathered}
$$

Wave conditions (16) for the first harmonic in the parabolic approximation for the magnetic field can be written as

$$
\left\{\begin{array}{c}
\frac{9}{2 L}+2 \frac{n_{\mathrm{e}}^{\prime}}{n_{\mathrm{e}}}-\frac{n_{\mathrm{p}}^{\prime}}{n_{\mathrm{p}}}>0, \\
\frac{4}{L} \frac{\pi \sqrt{2}}{\beta_{\mathrm{p}}}-\left(\frac{\varepsilon_{0_{\mathrm{p}}}^{\prime}}{\varepsilon_{0_{\mathrm{p}}}}+\frac{n_{\mathrm{p}}^{\prime}}{n_{\mathrm{p}}}\right)-2 \frac{\beta_{\mathrm{e}}}{\beta_{\mathrm{p}}}\left(\frac{\varepsilon_{0_{\mathrm{e}}}^{\prime}}{\varepsilon_{0_{\mathrm{e}}}}+\frac{n_{\mathrm{e}}^{\prime}}{n_{\mathrm{e}}}\right)>0,
\end{array}\right.
$$




$$
\left\{\begin{array}{c}
\frac{9}{2 L}+2 \frac{n_{\mathrm{e}}^{\prime}}{n_{\mathrm{e}}}-\frac{n_{\mathrm{p}}^{\prime}}{n_{\mathrm{p}}}<0, \\
\frac{4}{L} \frac{\pi \sqrt{2}}{\beta_{\mathrm{p}}}-\left(\frac{\varepsilon_{0_{\mathrm{p}}}^{\prime}}{\varepsilon_{0_{\mathrm{p}}}}+\frac{n_{\mathrm{p}}^{\prime}}{n_{\mathrm{p}}}\right)-2 \frac{\beta_{\mathrm{e}}}{\beta_{\mathrm{p}}}\left(\frac{\varepsilon_{0_{\mathrm{e}}}^{\prime}}{\varepsilon_{0_{\mathrm{e}}}}+\frac{n_{\mathrm{e}}^{\prime}}{n_{\mathrm{e}}}\right)<0 .
\end{array}\right.
$$
(19.1)

Instability condition (17) in this approximation is for

$$
\left(\frac{\omega_{0}}{\Omega_{\mathrm{d}_{\mathrm{e}}}}-S\right)\left(\frac{3}{L}+\frac{\varepsilon_{0_{\mathrm{e}}}^{\prime}}{\varepsilon_{0_{\mathrm{e}}}}\right)+\frac{n_{\mathrm{e}}^{\prime}}{n_{\mathrm{e}}}-\frac{3}{2} \frac{\varepsilon_{0_{\mathrm{e}}}^{\prime}}{\varepsilon_{0_{\mathrm{e}}}}<0
$$

and for (19.2)

$$
\left(\frac{\omega_{0}}{\Omega_{\mathrm{d}_{\mathrm{e}}}}-S\right)\left(\frac{3}{L}+\frac{\varepsilon_{0_{\mathrm{e}}}^{\prime}}{\varepsilon_{0_{\mathrm{e}}}}\right)+\frac{n_{\mathrm{e}}^{\prime}}{n_{\mathrm{e}}}-\frac{3}{2} \frac{\varepsilon_{0_{e}}}{\varepsilon_{0_{e}}}>0 .
$$

Thus, waves with frequencies much less than the electron magnetic drift frequency can propagate in the electron drift direction if condition (19.1) or (19.2) holds. This is possible even in the absence of plasma temperature or density gradients, i.e. only due to magnetic field inhomogeneity. At the same time, these waves can growth if the electron temperature and density gradients correspond to condition (20.1) for (19.1) and to (20.2) for (19.2).

The instability can exist for the Maxwell distribution of electron energy due to the temperature and density gradients $S=0 . \quad n_{\mathrm{e}}^{\prime} / n_{\mathrm{e}} \neq 0, \varepsilon_{0_{\mathrm{e}}}^{\prime} / \varepsilon_{0_{\mathrm{e}}} \neq 0$. Without these gradients, the instability can be caused by the inverted distribution of electron energy $S \neq 0$, $\left.n_{\mathrm{e}}^{\prime} / n_{\mathrm{e}}=0, \varepsilon_{0_{\mathrm{e}}}^{\prime} / \varepsilon_{0_{\mathrm{e}}}=0\right), \quad n_{\mathrm{p}}^{\prime} / n_{\mathrm{p}}=0, \quad \varepsilon_{0_{\mathrm{p}}}^{\prime} / \varepsilon_{0_{\mathrm{p}}}=0$. The greatest increase in the wave occurs with multidirectional radial gradients of electron temperature and density.

\section{EIGENFREQUENCY FAR EXCEEDING ELECTRON DRIFT FREQUENCY: $\omega / \Omega_{\mathrm{d}_{\mathrm{e}}} \gg 1$}

If we expand $Z\left(\sqrt{\omega / \Omega_{\mathrm{d}_{\mathrm{e}}}}\right)$ in the small parameter $\Omega_{\mathrm{d}_{\mathrm{e}}} / \omega \ll 1$ in $f_{\mathrm{e}}\left(\omega / \Omega_{\mathrm{d}_{\mathrm{e}}}\right)$ and neglect all terms such that the power of $\Omega_{\mathrm{d}_{\mathrm{e}}} / \omega$ is 2 or more, considering them small, we obtain the expression

$$
f_{\mathrm{e}}\left(\frac{\omega}{\Omega_{\mathrm{d}_{\mathrm{e}}}}\right)=\frac{\omega}{\Omega_{\mathrm{d}_{\mathrm{e}}}}\left(1-\frac{\omega_{\varepsilon_{\mathrm{e}}}^{*}}{\Omega_{\mathrm{d}_{\mathrm{e}}}}\right)-\left(\frac{\omega_{\varepsilon_{\mathrm{e}}}^{*}}{\Omega_{\mathrm{d}_{\mathrm{e}}}}+\frac{\omega_{n_{\mathrm{e}}}^{*}}{\Omega_{\mathrm{d}_{\mathrm{e}}}}\right) .
$$

Thus, in the case when the wave eigenfrequency is much greater than the electron drift frequency and the proton energy is much greater than the electron energy, the dispersion relation is expressed as:

$$
\begin{aligned}
& \frac{L_{\mathrm{b}_{\mathrm{p}}}}{\beta_{\mathrm{p}}} \Lambda_{N}=\frac{3}{4}\left(\frac{\omega_{\varepsilon_{\mathrm{p}}}^{*}}{\Omega_{\mathrm{d}_{\mathrm{p}}}}+\frac{\omega_{n_{\mathrm{p}}}^{*}}{\Omega_{\mathrm{d}_{\mathrm{p}}}}\right)-\frac{1}{2} a \frac{\omega}{\Omega_{\mathrm{d}_{\mathrm{e}}}}\left(\frac{3}{2}+\frac{\omega_{n_{\mathrm{p}}}^{*}}{\Omega_{\mathrm{d}_{\mathrm{p}}}}\right)+ \\
& +\frac{3}{2} \frac{\beta_{\mathrm{e}}}{\beta_{\mathrm{p}}}\left[\frac{\omega}{\Omega_{\mathrm{d}_{\mathrm{e}}}}\left(1-\frac{\omega_{\varepsilon_{\mathrm{e}}}^{*}}{\Omega_{\mathrm{d}_{\mathrm{e}}}}\right)-\left(\frac{\omega_{\varepsilon_{\mathrm{e}}}^{*}}{\Omega_{\mathrm{d}_{\mathrm{e}}}}+\frac{\omega_{n_{\mathrm{e}}}^{*}}{\Omega_{\mathrm{d}_{\mathrm{e}}}}\right)\right] .
\end{aligned}
$$

From (21) we derive the eigenfrequency expression: $\omega_{0}=\Omega_{d_{p}} \times$

$$
\times \frac{\frac{L_{\mathrm{b}_{\mathrm{p}}}}{\beta_{\mathrm{p}}} \Lambda_{N}-\frac{3}{4}\left(\frac{\omega_{\varepsilon_{\mathrm{p}}}^{*}}{\Omega_{\mathrm{d}_{\mathrm{p}}}}+\frac{\omega_{n_{\mathrm{p}}}^{*}}{\Omega_{\mathrm{d}_{\mathrm{p}}}}\right)+\frac{3}{2} \frac{\beta_{\mathrm{e}}}{\beta_{\mathrm{p}}}\left(\frac{\omega_{\varepsilon_{\mathrm{e}}}^{*}}{\Omega_{\mathrm{d}_{\mathrm{e}}}}+\frac{\omega_{n_{\mathrm{e}}}^{*}}{\Omega_{\mathrm{d}_{\mathrm{e}}}}\right)}{S+\frac{3}{4}-\frac{\omega_{\varepsilon_{\mathrm{e}}}^{*}}{\Omega_{\mathrm{d}_{\mathrm{e}}}}\left(S+\frac{3}{2}\right)-\frac{1}{2} \frac{\omega_{n_{\mathrm{p}}}^{*}}{\Omega_{\mathrm{d}_{\mathrm{p}}}}} .
$$

To find the increment, we again use the expansion in the small parameter and neglect the terms in the denominator, where the power of $\Omega_{\mathrm{d}_{\mathrm{e}}} / \omega$ is 2 and more:

$$
\begin{gathered}
\gamma=-\Omega_{\mathrm{d}_{\mathrm{e}}} \frac{\pi\left(\frac{\omega_{0}}{\Omega_{\mathrm{d}_{\mathrm{e}}}}\right)^{S+5 / 2} e^{-\frac{\omega_{0}}{\Omega_{\mathrm{d}_{\mathrm{e}}}}} \times}{\Gamma(S+3 / 2)} \times \\
\times \frac{\left[\left(\frac{\omega_{0}}{\Omega_{\mathrm{d}_{\mathrm{e}}}}-S\right)\left(1-\frac{\omega_{\varepsilon_{\mathrm{e}}}^{*}}{\Omega_{\mathrm{d}_{\mathrm{e}}}}\right)-\frac{\omega_{n_{\mathrm{e}}}^{*}}{\Omega_{\mathrm{d}_{\mathrm{e}}}}+\frac{3}{2} \frac{\omega_{\varepsilon_{\mathrm{e}}}^{*}}{\Omega_{\mathrm{d}_{\mathrm{e}}}}\right]}{S+\frac{3}{4}-\frac{\omega_{\varepsilon_{\mathrm{e}}}^{*}}{\Omega_{\mathrm{d}_{\mathrm{e}}}}\left(S+\frac{3}{2}\right)-\frac{1}{2} \frac{\omega_{n_{\mathrm{p}}}^{*}}{\Omega_{\mathrm{d}_{\mathrm{p}}}}} .
\end{gathered}
$$

Expression (22) shows that the wave, as for $\omega / \Omega_{\mathrm{d}_{\mathrm{e}}} \ll 1$, can exist in the absence of the gradients.

For the wave to exist, the following conditions should hold

$$
\left\{\begin{array}{c}
S+\frac{3}{4}-\frac{\omega_{\varepsilon_{\mathrm{e}}}^{*}}{\Omega_{\mathrm{d}_{\mathrm{e}}}}\left(S+\frac{3}{2}\right)-\frac{1}{2} \frac{\omega_{n_{\mathrm{p}}}^{*}}{\Omega_{\mathrm{d}_{\mathrm{p}}}}>0, \\
\frac{L_{\mathrm{b}_{\mathrm{p}}}}{\beta_{\mathrm{p}}} \Lambda_{N}-\frac{3}{4}\left(\frac{\omega_{\varepsilon_{\mathrm{p}}}^{*}}{\Omega_{\mathrm{d}_{\mathrm{p}}}}+\frac{\omega_{n_{\mathrm{p}}}^{*}}{\Omega_{\mathrm{d}_{\mathrm{p}}}}\right)+\frac{3}{2} \frac{\beta_{\mathrm{e}}}{\beta_{\mathrm{p}}}\left(\frac{\omega_{\varepsilon_{\mathrm{e}}}^{*}}{\Omega_{\mathrm{d}_{\mathrm{e}}}}+\frac{\omega_{n_{\mathrm{e}}}^{*}}{\Omega_{\mathrm{d}_{\mathrm{e}}}}\right)>0
\end{array}\right.
$$

or

$$
\left\{\begin{array}{c}
S+\frac{3}{4}-\frac{\omega_{\varepsilon_{\mathrm{e}}}^{*}}{\Omega_{\mathrm{d}_{\mathrm{e}}}}\left(S+\frac{3}{2}\right)-\frac{1}{2} \frac{\omega_{n_{\mathrm{p}}}^{*}}{\Omega_{\mathrm{d}_{\mathrm{p}}}}<0, \\
\frac{L_{\mathrm{b}_{\mathrm{p}}}}{\beta_{\mathrm{p}}} \Lambda_{N}-\frac{3}{4}\left(\frac{\omega_{\varepsilon_{\mathrm{p}}}^{*}}{\Omega_{\mathrm{d}_{\mathrm{p}}}}+\frac{\omega_{n_{\mathrm{p}}}^{*}}{\Omega_{\mathrm{d}_{\mathrm{p}}}}\right)+\frac{3}{2} \frac{\beta_{\mathrm{e}}}{\beta_{\mathrm{p}}}\left(\frac{\omega_{\varepsilon_{\mathrm{e}}}^{*}}{\Omega_{\mathrm{d}_{\mathrm{e}}}}+\frac{\omega_{n_{\mathrm{e}}}^{*}}{\Omega_{\mathrm{d}_{\mathrm{e}}}}\right)<0 .
\end{array}\right.
$$

The instability condition from (23) for (24.1) is

$\left(\frac{\omega_{0}}{\Omega_{\mathrm{d}_{\mathrm{e}}}}-S\right)\left(1-\frac{\omega_{\varepsilon_{\mathrm{e}}}^{*}}{\Omega_{\mathrm{d}_{\mathrm{e}}}}\right)-\frac{\omega_{n_{\mathrm{e}}}^{*}}{\Omega_{\mathrm{d}_{\mathrm{e}}}}+\frac{3}{2} \frac{\omega_{\varepsilon_{\mathrm{e}}}^{*}}{\Omega_{\mathrm{d}_{\mathrm{e}}}}<0$ 
and for (24.2),

$$
\left(\frac{\omega_{0}}{\Omega_{\mathrm{d}_{\mathrm{e}}}}-S\right)\left(1-\frac{\omega_{\varepsilon_{\mathrm{e}}}^{*}}{\Omega_{\mathrm{d}_{\mathrm{e}}}}\right)-\frac{\omega_{n_{\mathrm{e}}}^{*}}{\Omega_{\mathrm{d}_{\mathrm{e}}}}+\frac{3}{2} \frac{\omega_{\varepsilon_{\mathrm{e}}}^{*}}{\Omega_{\mathrm{d}_{\mathrm{e}}}}>0 .
$$

In the parabolic approximation of magnetic field for the first harmonic $N=1$, the wave existence conditions can be written as

$$
\left\{\begin{array}{c}
\frac{3}{L}\left(S+\frac{3}{4}\right)+\frac{\varepsilon_{0_{\mathrm{e}}}^{\prime}}{\varepsilon_{0_{\mathrm{e}}}}\left(S+\frac{3}{2}\right)-\frac{1}{2} \frac{n_{\mathrm{p}}^{\prime}}{n_{\mathrm{p}}}>0, \\
\frac{4}{L} \frac{\pi \sqrt{2}}{\beta_{\mathrm{p}}}-\left(\frac{\varepsilon_{0_{\mathrm{p}}}^{\prime}}{\varepsilon_{0_{\mathrm{p}}}}+\frac{n_{\mathrm{p}}^{\prime}}{n_{\mathrm{p}}}\right)-2 \frac{\beta_{\mathrm{e}}}{\beta_{\mathrm{p}}}\left(\frac{\varepsilon_{0_{\mathrm{e}}}^{\prime}}{\varepsilon_{0_{\mathrm{e}}}}+\frac{n_{\mathrm{e}}^{\prime}}{n_{\mathrm{e}}}\right)>0
\end{array}\right.
$$

or

$$
\left\{\begin{array}{c}
\frac{3}{L}\left(S+\frac{3}{4}\right)+\frac{\varepsilon_{0_{\mathrm{e}}}^{\prime}}{\varepsilon_{0_{\mathrm{e}}}}\left(S+\frac{3}{2}\right)-\frac{1}{2} \frac{n_{\mathrm{p}}^{\prime}}{n_{\mathrm{p}}}<0, \\
\frac{4}{L} \frac{\pi \sqrt{2}}{\beta_{\mathrm{p}}}-\left(\frac{\varepsilon_{0_{\mathrm{p}}}^{\prime}}{\varepsilon_{0_{\mathrm{p}}}}+\frac{n_{\mathrm{p}}^{\prime}}{n_{\mathrm{p}}}\right)-2 \frac{\beta_{\mathrm{e}}}{\beta_{\mathrm{p}}}\left(\frac{\varepsilon_{0_{\mathrm{e}}}^{\prime}}{\varepsilon_{0_{\mathrm{e}}}}+\frac{n_{\mathrm{e}}^{\prime}}{n_{\mathrm{e}}}\right)<0 .
\end{array}\right.
$$

The instability condition for (26.1) is

$$
\left(\frac{\omega_{0}}{\Omega_{\mathrm{d}_{\mathrm{e}}}}-S\right)\left(\frac{3}{L}+\frac{\varepsilon_{0_{\mathrm{e}}}^{\prime}}{\varepsilon_{0_{\mathrm{e}}}}\right)+\frac{n_{\mathrm{e}}^{\prime}}{n_{\mathrm{e}}}-\frac{3}{2} \frac{\varepsilon_{0_{\mathrm{e}}}^{\prime}}{\varepsilon_{0_{\mathrm{e}}}}<0
$$

and for (26.2),

$$
\left(\frac{\omega_{0}}{\Omega_{\mathrm{d}_{\mathrm{e}}}}-S\right)\left(\frac{3}{L}+\frac{\varepsilon_{0_{\mathrm{e}}}^{\prime}}{\varepsilon_{0_{\mathrm{e}}}}\right)+\frac{n_{\mathrm{e}}^{\prime}}{n_{\mathrm{e}}}-\frac{3}{2} \frac{\varepsilon_{0_{\mathrm{e}}}^{\prime}}{\varepsilon_{0_{\mathrm{e}}}}>0 .
$$

Waves with frequencies much greater than the electron magnetic drift frequency $\omega / \Omega_{d_{e}} \gg 1$ exist when the proton temperature and density gradients satisfy condition (26.1) or (26.2). As for $\omega / \Omega_{\mathrm{d}_{\mathrm{e}}} \ll 1$, the wave can exist in the absence of such gradients. The instability develops if the electron temperature and density gradients meet condition (27.1) for (26.1) and (27.2) for (26.2).

\section{RESULTS OF NUMERICAL CALCULATIONS}

To make numerical calculations, we take $\beta_{\mathrm{e}} / \beta_{\mathrm{p}}=0.1$. We plot (Figures 1 and 2) exact solutions of dispersion relation (11) represented as

$$
f\left(\omega_{N}\right) \equiv f_{\mathrm{p}}\left(a \omega_{N}\right)+\frac{3}{2} \frac{\beta_{\mathrm{e}}}{\beta_{\mathrm{p}}} f_{\mathrm{e}}\left(\omega_{N}\right)=\frac{L_{\mathrm{b}_{\mathrm{p}}}}{\beta_{\mathrm{p}}} \Lambda_{N},
$$

for various parameters of plasma gradients and its inversion. The wave can exist at $\omega_{N}$ when $\operatorname{Re} f\left(\omega_{N}\right)>0$ and $\operatorname{Im} f\left(\omega_{N}\right)=0$ since $L_{\mathrm{b}_{\mathrm{p}}} \Lambda_{N} / \beta_{\mathrm{p}}$ is a positive and real value. On the plots, the positive $\operatorname{Re} f(\omega)$ values are marked with red color; negative ones, for which there are no solutions, with blue color. Isolines in the region of positive values correspond to $\operatorname{Re} f(\omega)=L_{\mathrm{b}_{\mathrm{p}}} \Lambda_{N} / \beta_{\mathrm{p}}$. The thick line indicates $\operatorname{Im} f(\omega)=0$. Thus, to solutions of dispersion relation (28) correspond intersection points of isolines $\operatorname{Re} f(\omega)$ in the region of positive values with the line $\operatorname{Im} f(\omega)=0$.

Figure 1, $a$ shows that the line $\operatorname{Im} f(\omega)=0$ intersects the isolines $L_{\mathrm{b}_{\mathrm{p}}} \Lambda_{N} / \beta_{\mathrm{p}}$, where $\operatorname{Im}\left(\omega / \Omega_{\mathrm{d}_{\mathrm{e}}}\right)<0$. We can conclude that in the absence of gradients and inverted electron energy distribution, the instability cannot exist. The same conclusion can be drawn if we substitute the corresponding values $\quad \varepsilon_{0_{\mathrm{p}}}^{\prime} / \varepsilon_{0_{\mathrm{p}}}=0, \quad n_{\mathrm{p}}^{\prime} / n_{\mathrm{p}}=0$, $\varepsilon_{0_{\mathrm{e}}}^{\prime} / \varepsilon_{0_{\mathrm{e}}}=0, n_{\mathrm{e}}^{\prime} / n_{\mathrm{e}}=0, S=0$ and analyze Expressions (19.1), (26.1) and (20.1), (27.1). As may be inferred from (19.1), (26.1), the wave can exist; however, from (20.1), (27.1) it follows that the instability is not realized because inequalities (20.1) and (27.1) fail. If we add the inverted distribution (Figure 1, b), we get a situation, where the line $\operatorname{Im} f(\mathrm{w})=0$ intersects the isolines $L_{\mathrm{b}_{\mathrm{p}}} \Lambda_{N} / \beta_{\mathrm{p}}$ in the region with $\operatorname{Im}\left(\omega / \Omega_{\mathrm{d}_{\mathrm{e}}}\right)>0$ and $\operatorname{Re} f\left(\omega_{N}\right)>0$, i.e. all conditions, under which the plasma instability occurs, hold. If we substitute the respective values $\quad \varepsilon_{0_{\mathrm{p}}}^{\prime} / \varepsilon_{0_{\mathrm{p}}}=0, \quad n_{\mathrm{p}}^{\prime} / n_{\mathrm{p}}=0, \quad \varepsilon_{0_{\mathrm{e}}}^{\prime} / \varepsilon_{0_{\mathrm{e}}}=0$, $n_{\mathrm{e}}^{\prime} / n_{\mathrm{e}}=0, S=1$ in (19.1) and (20.1), the inequalities hold, and hence the wave can be increased.

The instability can also exist for the Maxwell distribution of electron energies (Figure 2,a), but in the presence of particle temperature and density gradients, the inverted distribution (Figure 2,b) strengthens the instability. Note that for the numerical calculations we use the formula without approximation with respect to $\omega / \Omega_{d_{e}}$. The Figures show that the instability has a maximum increment when the eigenfrequency of the drift-compressional mode is close to the electron drift frequency.

Also note that since plasma in Earth's magnetosphere is usually cold, i.e. $\beta>1$, the above results only illustrate that the instability can exist because we consider hot plasma with $\beta<1$. At small $\beta$ values, even for the fundamental harmonic $L_{\mathrm{b}_{\mathrm{p}}} \Lambda_{N} \approx 4.4$ if $\quad N=1, \quad L_{\mathrm{b}_{\mathrm{p}}} \Lambda_{N} / \beta_{\mathrm{p}} \gg 1 ; \quad$ therefore, $\omega_{0} / \Omega_{\mathrm{d}_{\mathrm{e}}} \gg 1$, is the closest parameter to real magnetospheric plasma parameters (asymptotic expressions (22) and (23)). As an example, we find the fundamental frequency of the drift-compressional mode for $L=6.6 R_{\mathrm{E}}$, assuming, for simplicity, that temperature and density gradients are small. Suppose that the electron energy at the maximum of the inverted distribution $\varepsilon_{\mathrm{e}_{\max }}=10 \mathrm{keV}$, the azimuthal wave number $k_{2}=70$ and the parameters $\beta_{\mathrm{e} /} \beta_{\mathrm{p}}=0.1, \beta_{\mathrm{p}}=0.5$. 

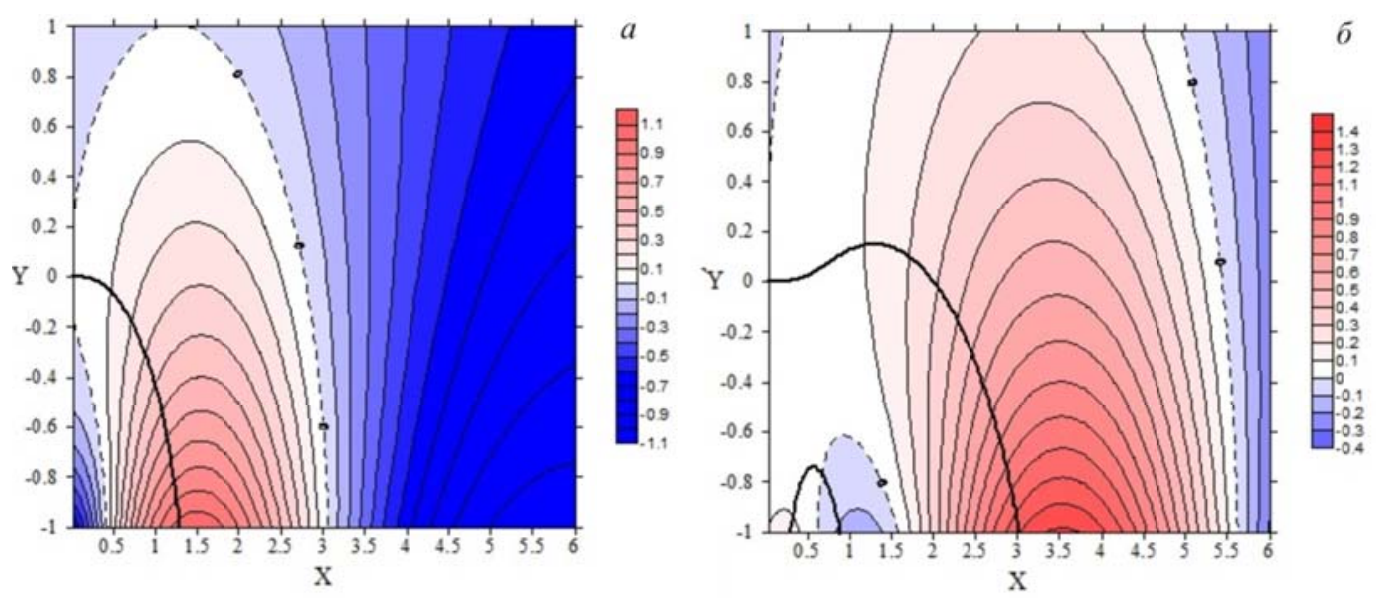

Figure 1. Solutions of dispersion relation (28) for various plasma parameters (in the parabolic approximation for the magnetic field): $\varepsilon_{0_{\mathrm{p}}}^{\prime} / \varepsilon_{0_{\mathrm{p}}}=0, \quad n_{\mathrm{p}}^{\prime} / n_{\mathrm{p}}=0, \quad \varepsilon_{0_{\mathrm{e}}}^{\prime} / \varepsilon_{0_{\mathrm{e}}}=0, \quad n_{\mathrm{e}}^{\prime} / n_{\mathrm{e}}=0, \quad S=0 \quad(a) ; \quad \varepsilon_{0_{\mathrm{p}}}^{\prime} / \varepsilon_{0_{\mathrm{p}}}=0, \quad n_{\mathrm{p}}^{\prime} / n_{\mathrm{p}}=0, \quad \varepsilon_{0_{\mathrm{e}}}^{\prime} / \varepsilon_{0_{\mathrm{e}}}=0$, $n_{\mathrm{e}}^{\prime} / n_{\mathrm{e}}=0, S=1(b)$. Here, $X=\operatorname{Re}\left(\omega / \Omega_{\mathrm{d}_{\mathrm{e}}}\right), Y=\operatorname{Im}\left(\omega / \Omega_{\mathrm{d}_{\mathrm{e}}}\right)$, the isolines in the region of positive values correspond to $L_{\mathrm{b}_{\mathrm{p}}} \Lambda_{\mathrm{N}} / \beta_{\mathrm{p}}$ ; the points of their intersection with the bold line, the solutions $\omega_{N} / \Omega_{d_{e}}$. The step of the change in $L_{b_{p}} \Lambda_{N} / \beta_{p}$ is represented to the right of each plot
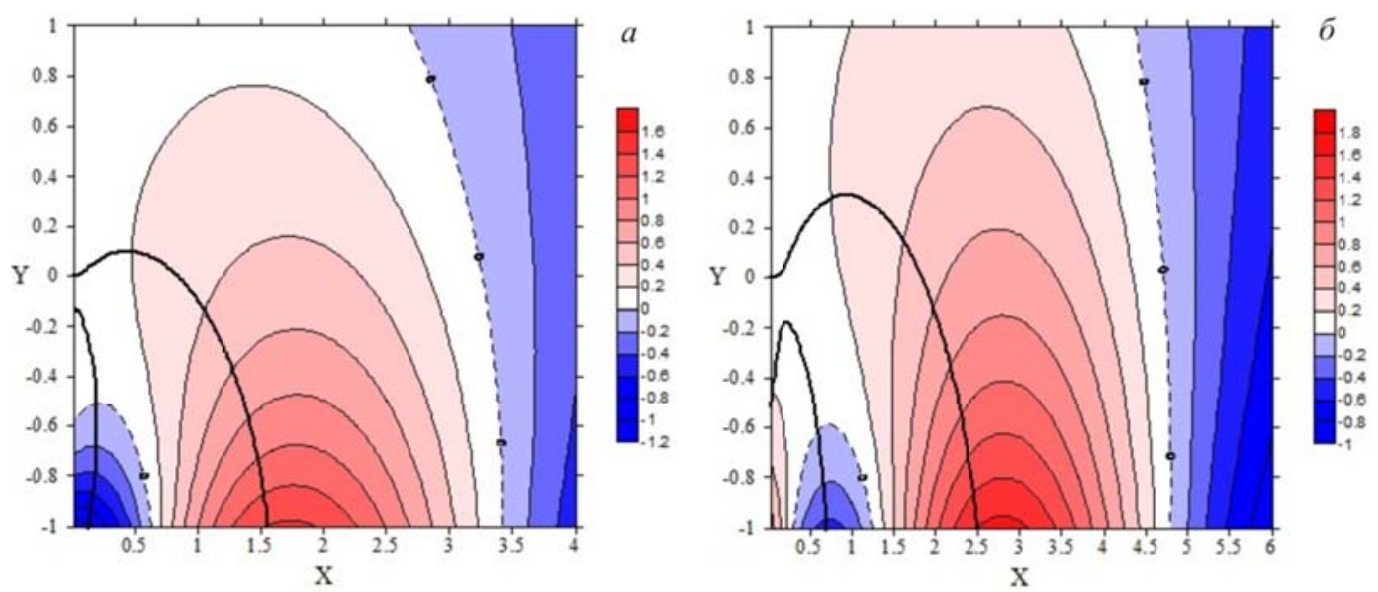

Figure 2. Same as in Figure 1 for the following plasma parameters: $\varepsilon_{0_{\mathrm{p}}}^{\prime} / \varepsilon_{0_{\mathrm{p}}}=0.3 L^{-1}, \quad n_{\mathrm{p}}^{\prime} / n_{\mathrm{p}}=0.3 L^{-1}, \quad \varepsilon_{0_{\mathrm{e}}}^{\prime} / \varepsilon_{0_{\mathrm{e}}}=1.5 L^{-1}$, $n_{\mathrm{e}}^{\prime} / n_{\mathrm{e}}=-1.5 L^{-1}, S=0(a) ; \varepsilon_{0_{\mathrm{p}}}^{\prime} / \varepsilon_{0_{\mathrm{p}}}=0.3 L^{-1}, n_{\mathrm{p}}^{\prime} / n_{\mathrm{p}}=0.3 L^{-1}, \quad \varepsilon_{0_{\mathrm{e}}}^{\prime} / \varepsilon_{0_{\mathrm{e}}}=1.5 L^{-1^{\mathrm{p}}}, \quad n_{\mathrm{e}}^{\prime \mathrm{p}} / n_{\mathrm{e}}=-1.5 L^{-1}, S=\mathbb{1} \quad(b)$

Neglecting the gradients, we get that the instability arises when $S>\omega_{0} / \Omega_{\mathrm{d}_{\mathrm{e}}}$. For the chosen plasma parameters it is easy to show that $\omega_{0} / \Omega_{\mathrm{d}_{\mathrm{e}}} \gg 1$; therefore, we determine the frequency $\omega_{0}$ and the instability increment $\gamma$, using Expressions (22) and (23) respectively. For small gradients, we obtain

$$
\begin{aligned}
& \omega_{0}=\Omega_{\mathrm{d}_{\mathrm{p}}}\left(\frac{L_{\mathrm{b}_{\mathrm{p}}}}{\beta_{\mathrm{p}}} \Lambda_{\mathrm{N}}\right) /\left(S+\frac{3}{4}\right), \\
& \gamma=\Omega_{\mathrm{d}_{\mathrm{e}}} \frac{\pi\left(\frac{\omega_{0}}{\Omega_{\mathrm{d}_{\mathrm{e}}}}\right)^{S+5 / 2} e^{-\frac{\omega_{0}}{\Omega_{\mathrm{d}_{\mathrm{e}}}}} \frac{\left(S-\frac{\omega_{0}}{\Omega_{\mathrm{d}_{\mathrm{e}}}}\right)}{\Gamma(S+3 / 2)} .}{\left(S+\frac{3}{4}\right)} .
\end{aligned}
$$

The instability occurs if in model inverted electron distribution (2) $S>59$. For the calculations, we put $S=60$ (Figure 3). In this case, $f_{0}=\mathbf{1 . 6} \mathrm{mHz}$, the increment $\gamma=4.8 \cdot 10^{-5} s^{-1}$, and $\gamma / f_{0}=3 \cdot 10^{-2}$. Note that across the given L-shell with the Alfvén velocity $V_{\mathrm{A}}=1000 \mathrm{~km} / \mathrm{s}$, the fundamental frequency of Alfvén resonance is $~ 7.7$ $\mathrm{mHz}$. Thus, the frequency of the drift-compressional wave turns out to be lower than the Alfvén resonance frequency. At smaller values of the azimuthal wave number $k_{2}$, the drift-compressional wave frequency is even less as being directly proportional to $k_{2}$. The resulting ratio of the increment to the eigenfrequency is close to the values obtained in [Hughes et al., 1978] for Alfvén waves, generated by the bounce drift instability, and to the damping decrement on the ionosphere. 


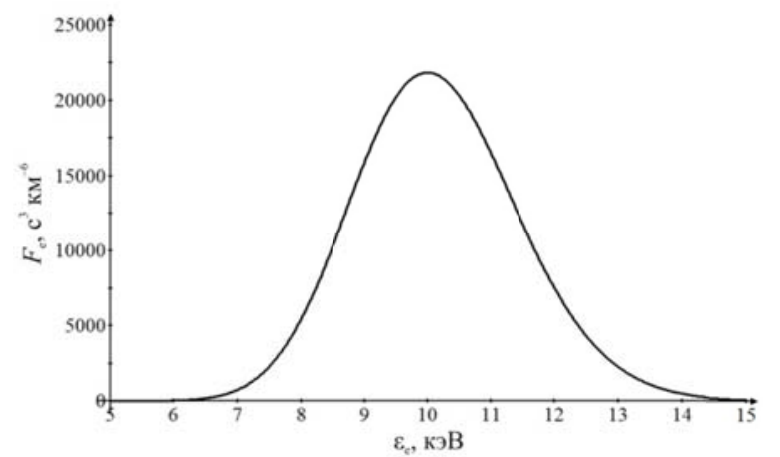

Figure 3. Model distribution function of hot electrons (2) with $\varepsilon_{\mathrm{e}_{\max }}=10 \mathrm{keV}, \beta_{\mathrm{e}}=0.05, S=60$

\section{CONCLUSION}

The results allow us to conclude that driftcompressional waves can propagate not only in the proton drift direction (to the west) [Crabtree et al., 2003, Klimushkin, Mager, 2011, Mager et al., 2013], but also in the electron drift direction (to the east). These waves can also exist in the absence of plasma temperature and density gradients. In this case, the wave phase velocity should be less than the mean particle magnetic drift velocity in the bump. Propagating in the electron drift direction, these waves can be increased due to resonant interaction with electrons, i.e due to drift instability. This instability may develop at certain electron and proton temperature and density gradients or because of the inverted electron energy distribution.

For the magnetic shell $L=6.6 R_{\mathrm{E}}$, we have demonstrated that frequencies of drift-compressional waves can be lower than eigenfrequencies of magnetic tube oscillations: at $V_{\mathrm{A}}=1000 \mathrm{~km} / \mathrm{s}$, the fundamental frequency of Alfvén resonance is $7.7 \mathrm{mHz}$, and the drift-compressional wave frequency with the azimuthal wave number $k_{2}=70$ is $1.6 \mathrm{mHz}$. Because of the resonant interaction of the wave with hot electrons, its amplitude increases with an increment of 0.03 to the frequency.

Our results can be helpful in interpreting observations of wave phenomena with frequencies in the range of Pc5 geomagnetic pulsations and below. For example, in [James et al., 2013], SuperDARN data have been used to statistically analyze ULF oscillations occurring during substorm activity; it has been shown that in addition to the waves propagating in the proton drift direction (to the west), there are waves running in the electron drift direction (to the east). In this case, periods of some of these waves considerably exceed those of Pc5 pulsations. Most likely these waves are not Alfvén - they are probably drift-compressional, running in the electron drift direction, and increased due to resonant interaction with energetic electrons injected into the magnetosphere during substorms.

The work was supported by RFBR grant No. 16-0500254a.

\section{REFERENCES}

Allan W., Poulter E.M., Nielsen E. STARE observations of a Pc5 pulsation with large azimuthal wave number. $J$. Geophys. Res. 1982, vol. 87, pp. 6163-6172. DOI: 10.1029/JA087iA08 p06163.

Barfield J.N., McPherron R.L. Statistical characteristics of storm-associated Pc5 micropulsations observed at the synchronous equatorial orbit. J. Geophys. Res. 1972, vol. 77, pp. 4720-4733. DOI: 10.1029/JA077i025p04720.

Chelpanov M.A., Mager P.N., Klimushkin D.Y., Berngardt O.L., Mager O.V. Experimental evidence of drift compressional waves in the magnetosphere: an Ekaterinburg coherent decameter radar case study. J. Geophys. Res. Space Phys. 2016, vol. 121, pp. 1315-1326. DOI: 10.1002/2015JA022155.

Chen L., Hasegawa A. A theory of long period magnetic pulsation. 1. Steady state excitation of a field line resonance $J$. Geophys. Res. 1974, vol. 79, pp. 1024-1032. DOI: 10.1029/JA079i 007p01024.

Chen L., Hasegawa A. Kinetic theory of geomagnetic pulsations. 1. Internal excitations by energetic particles. $J$. Geophys. Res. 1991, vol. 96, pp. 1503-1512. DOI: 10.1029/ 90JA02346.

Crabtree C., Horton W., Wong H.V., van Dam J.W. Bounce-averaged stability of compressional modes in geotail flux tubes. J. Geophys. Res. 2003, vol. 108, p. 1084. DOI: 10.1029/2002JA009555.

Fenrich F.R., Samson J.C., Sofko G., Greenwald R.A. ULF high- and low-m field line resonances observed with the Super Dual Auroral Radar Network. J. Geophys. Res. 1995, vol. 100, pp. 21,535-21,548. DOI: 10.1029/95JA02024.

Hamlin D.A., Karplus R., Vik. R.C., Watson K.M. Mirror and azimuthal drift frequencies for geomagnetically trapped particles. J. Geophys. Res. 1961, vol. 66, no. 1, pp. 1-4. DOI: 10.1029/ JZ066i001p00001.

Higuchi T., Kokubun S. Waveform and polarization of compressional Pc5 waves at geosynchronous orbit. $J$. Geophys. Res. 1988, vol. 93, pp. 14,433-14,443. DOI: 10.1029/ JA093iA12p14433.

Hughes W.J., Southwood D.J., Mauk B., McPherron R.L., Barfield J.N. Alfvén waves generated by an inverted plasma energy distribution., Nature. 1978, vol. 275, pp. 43-45. DOI: 10.1038/ 275043a0.

Hurricane O.A., Pellat R., Coroniti F.V. The kinetic response of a stochastic plasma to low frequency perturbations. J. Geophys. Res. 1994, vol. 21, no. 4, pp. 253256. DOI: $10.1029 / 93$ GL03533.

James M.K., Yeoman T.K., Mager P.N., Klimushkin D.Y. The spatio-temporal characteristics of ULF waves driven by substorm injected particles. J. Geophys. Res. Space Phys. 2013, vol. 118, pp. 1737-1749. DOI: 10.1002/jgra.50131.

Klimushkin D.Y., Mager P'.N. Spatial structure and stability of coupled Alfvén and drift compressional modes in non-uniform magnetosphere: gyrokinetic treatment. Planet. Space Sci. 2011, vol. 59, pp. 1613-1620. DOI: 10.1016/j.pss.2011.07.010.

Klimushkin D.Y., Mager P.N., Pilipenko V.A. On the ballooning instability of the coupled Alfven and drift compressional modes. Earth, Planets and Space. 2012, vol. 64, pp. 777-781. DOI: 10.5047/eps.2012.04.002.

Kremser G., Korth A., Fejer J.A., Wilken B., Gurevich A.V., Amata E. Observations of quasi-periodic flux variations of energetic ions and electrons associated with Pc5 
geomagnetic pulsations. J. Geophys. Res. 1981, vol. 86, pp. 3345-3356. DOI: 10.1029/JA086iA05p03345.

Leonovich A.S., Mazur V.A. Resonance excitation of standing Alfvén waves in an axisymmetric magnetosphere (Monochromatic oscillations). Planet. Space Sci. 1989, vol. 37, pp. 1095-1108.

Leonovich A.S., Mazur V.A. A theory of transverse smallscale standing Alfvén waves in an axially symmetric magnetosphere. Planet. Space Sci. 1993, vol. 41, pp. 697-717. DOI: 10.1016/0032-0633(93)90055-7.

Mager P.N., Berngardt O.I., Klimushkin D.Y., Zolotukhina N.A., Mager O.V. First results of the highresolution multibeam ULF wave experiment at the Ekaterinburg SuperDARN radar: ionospheric signatures of coupled poloidal Alfvén and drift-compressional modes. $J$. Atmos. Solar-Terr. Phys. 2015, vol. 130-131, pp. 112-126. DOI: $10.1016 /$ j.jastp.2015.05.017.

Mager P.N., Klimushkin D.Y., Kostarev D.V. Driftcompressional modes generated by inverted plasma distributions in the magnetosphere. J. Geophys. Res. Space Phys. 2013, vol. 118, pp. 4915-4923. DOI: 10.1002 /jgra.50471.
Pokhotelov O.A., Onishchenko O.G., Balikhin M.A., Treumann R.A., Pavlenko V. P. Drift mirror instability in space plasmas. 2. Nonzero electron temperature effects. $J$. Geophys. Res. 2001, vol. 106, pp. 13,237-13,246.

Southwood D.J. Some features of field line resonances in the magnetosphere. Planet. Space Sci. 1974, vol. 22, pp. 483491. DOI: 10.1016/0032-0633(74)90078-6.

Walker A.D.M. Magnetohydrodynamic Waves in Geospace. The Theory of ULF Waves and Their Interaction with Energetic Particles in the Solar-Terrestrial Environment. 2005, pp. 503-506.

Yeoman T.K., Tian M., Lester M., Jones T.B. A study of Pc5 hydromagnetic waves with equatorward phase propagation. Planet. Space Sci. 1992, vol. 40, pp. 797-810. DOI: 10.1016/ 0032-0633(92)90108-Z.

How to cite this article

Kostarev D.V., Mager P.N. Drift-compression waves propagating in the drift direction of energetic electrons in the magnetosphere. Solar-Terrestrial Physics. 2017. Vol. 3, iss. 3. P. 18-27. DOI: $10.12737 /$ stp-33201703 